\title{
Génétique des anhydrases carboniques
}

Le groupe des anhydrases carboniques (carbonate dehydratase, carbonate hydroxylyase) est constitué de métalloenzymes à zinc. Elles catalysent l'hydratation réversible du $\mathrm{CO}_{2} \quad\left(\mathrm{CO}_{2} \rightleftharpoons\right.$ $\mathrm{HCO}_{3}{ }^{-}+\mathrm{H}^{+}$) et possèdent une activité extrêmement élevée. Elles peuvent également exercer une action d'estérase.

$\mathrm{Ce}$ groupe comprend au moins trois isozymes bien définies, et peut-être deux autres moins bien identifiées. Leur monomère a une taille voisine de 29000 daltons. Les isozymes CA I et CA II. La séparation des deux premières anhydrases carboniques CA I et CA II date de 1960 . Elles different par leur structure, leurs propriétés biochimiques, leur distribution et leur importance pathologique.

Leur séquence d'acides aminés présente environ $50 \%$ d'homologie. Elles n'ont pas de réaction immunologique croisée. La CA II possède une activité par molécule plus forte que la CA I tant pour son action hydratasique que pour la plupart des réactions estérasiques. L'inhibition par les sulfamides, caractère commun à l'ensemble du groupe, est également plus marquée pour la CA II. La distribution est très différente : la CA I est concentrée dans les globules rouges et son taux est très faible dans les tissus ; au contraire la CA II est présente, en plus des globules rouges, dans de nombreux tissus. Au cours de la croissance, les taux des deux isozymes dans les hématies est très bas chez le fotus et à la naissance et n'atteint le niveau adulte qu'à la fin de la première année.

La possibilité de séparer les deux isozymes par inhibition différentielle et par immunologie a permis de distinguer des déficits isolés en chacune des anhydrases carboniques; les conséquences en sont très différentes. Le déficit en CA I, observé il y a dix ans [1] $\mathrm{m} / \mathrm{s} n^{\circ} 4$ vol. 3, avril 87 ne provoquait aucun trouble clinique. Au contraire, le déficit en CA II s'accompagne d'un syndrome d'ostéopétrose (ossification excessive des os) avec acidose tubulaire rénale, d'hérédité autosomique récessive ; c'est la recherche de la CA II qui permet de la distinguer des autres formes d'ostéopétrose [2]. L'obtention de clones d'ADN complémentaire, conjuguée à l'emploi d'hybrides somatiques homme-souris, a assuré la localisation du gène de la CA II sur le chromosome 8. Comme des travaux antérieurs de génétique classique avaient montré une liaison étroite entre la CA I et la CA II, ces résultats localisaient les deux gènes des isozymes classiques sur le chromosome 8 [3], résultat confirmé par l'emploi d'une sonde d'ADNc correspondant à la CA I [4]. Anhydrase carbonique III (CA III). Connue depuis une dizaine d'années, cette isozyme differe des deux autres par son activité plus faible vis-à-vis du substrat principal, sa résistance plus grande aux sulfamides, sa mobilité électrophorétique différente, et surtout sa distribution; on l'a d'abord crue confinée aux muscles, et même à un certain type de muscle : très abondante dans les muscles lents comme le soléaire, elle est presque absente des muscles rapides et du muscle cardiaque. Elle est en fait présente, mais à taux faibles, dans d'autres tissus, et à taux élevés dans le foie du rat mâle, où sa régulation semble soumise aux androgènes. Sa prédominance musculaire chez l'homme a fait proposer son élévation dans le plasma comme test capable de concurrencer la créatine kinase dans le diagnostic de la myopathie de Duchenne.

L'intérêt qui s'attache à la CA III a fait un bond avec l'obtention récente d'un ADNc humain simultanément dans deux laboratoires $[5,6]$. L'utilisation d'hybri- des somatiques a rapidement conduit à la localisation du gène sur le chromosome 8 [6].

Les trois gènes qui codent pour les trois isozymes caractérisées de la CA sont dont situés sur le même chromosome. Bien que ce ne soit pas démontré pour la CA III, il est raisonnable de penser qu'ils sont groupés comme, par exemple, les gènes de la $\beta$-globine sur le chromosome 11 et ceux du fibrinogène sur le chromosome 4 . Sur le plan de l'évolution, l'apparition des anhydrases carboniques est certainement très ancienne, car on les retrouve chez des bactéries et des plantes comme dans toute l'échelle animale. La comparaison des séquences des isozymes I et II conduit à faire remonter leur séparation, par duplication d'un gène ancestral, à environ 300 millions d'années, la CA III se séparant de la CA II à une date ultérieure. Quant aux interrégulations entre les isozymes, s'il en existe, elles restent encore complètement inconnues.

J.-C. D.

1. Kendall AC, Tashian RE. Erythrocyte carbonic anhydrase I (CA I) : inherited deficiency in humans. Science $1977 ; 197: 471-2$. 2. Sly WS, Hewett-Emmett D, Whyte MP, Yu YSL, Tashian RE. Carbonic anhydrase II deficiency identified as the primary defect in the autosomal recessive syndrome of osteopetrosis with renal tubular acidosis and cerebral calcification. Proc Natl Acad Sci USA 1983; 80 : 2752-6.

3. Venta PJ, Shows TB, Curtis PJ, Tashian RE. Polymorphic gene for human carbonic anhydrase II : a molecular disease marker located on chromosome 8. Proc Natl Sci USA 1983 ; $80: 4437-40$.

4. Edwards YH, Barlow JH, Konialis CP, Povey S, Butterworth PHW. Assignment of the gene determining human carbonic anhydrase, CA I, to chromosome 8. Ann Hum Genet 1986 ; $50: 123-9$.

5. Lloyd J, McMillan S, Hopkinson D, Edwards YH. Nucleotide sequence and derived aminoacid sequence of a cDNA encoding human muscle carbonic anhydrase. Gene 1986 ; 41: 233-9.

6. Wade R, Gunning P, Eddy R, Shows T, Kedes L. Nucleotide sequence, tissue-specific expression, and chromosome location of human carbonic anhydrase III. Proc Natl Acad Sci USA $1986 ; 83: 9571-5$.

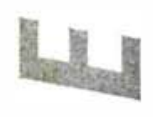

